\title{
Extracorporeal shockwave therapy enhances peripheral nerve remyelination and gait function in a crush model
}

\author{
Hyun Jung Park 1,B, , Jinpyo Hong ${ }^{1, B}$, Yibo Piao, ${ }^{1, B}$, Hyo Jung Shin ${ }^{1,2, B}$, Se Jeong Lee ${ }^{3, B}$, \\ Im Joo Rhyu ${ }^{3, B}$, Min-Hee Yi, ${ }^{4, A}$, , Jinhyun Kim ${ }^{5, A}$, Dong Woon Kim ${ }^{1,2, A, E}$, Jaewon Beom ${ }^{6, A, C, D, F}$ \\ 1 Department of Anatomy, Brain Research Institute, Chungnam National University School of Medicine, Daejeon, South Korea \\ 2 Department of Medical Science, Chungnam National University School of Medicine, Daejeon, South Korea \\ ${ }^{3}$ Department of Anatomy, Korea University College of Medicine, Seoul, South Korea \\ ${ }^{4}$ Department of Neurology, Mayo Clinic, Rochester, USA \\ ${ }^{5}$ Department of Internal Medicine, Chungnam National University Hospital, Chungnam National University School of Medicine, Daejeon, South Korea \\ ${ }^{6}$ Department of Rehabilitation Medicine, Seoul National University Bundang Hospital, Seongnam, South Korea \\ A - research concept and design; B - collection and/or assembly of data; $C$ - data analysis and interpretation; \\ $D$ - writing the article; $E$ - critical revision of the article; $F$ - final approval of the article
}

Address for correspondence

Jaewon Beom

E-mail: powe5@snubh.org

Funding sources

The Basic Science Research Program through the National Research Foundation of Korea (NRF) (grants No. 2016R1D1A1B03933919 and

No. 2016R1A2B4009409) funded this research.

Conflict of interest

None declared

Received on April 8, 2019

Accepted on May 8, 2020

Published online on July 31,2020

Cite as

Park HJ, Hong J, Piao Y, et al. Extracorporeal shockwave therapy enhances peripheral nerve remyelination and gait function in a crush model. Adv Clin Exp Med. 2020;29(7): 819-824. doi:10.17219/acem/122177

DOI

10.17219/acem/122177

Copyright

Copyright by Author(s)

This is an article distributed under the terms of the

Creative Commons Attribution 3.0 Unported (CC BY 3.0)

(https://creativecommons.org/licenses/by/3.0/)

\section{Abstract}

Background. Conservative treatment, such as electrical stimulation and steroid injection, have been employed in an attempt to improve symptoms after peripheral nerve injury, without significant success. Although non-invasive and safe extracorporeal shockwave therapy (ESWT) can be a practical alternative, the therapeutic effects of ESWT on peripheral nerve remyelination has not been established.

Objectives. To investigate the effects of ESWT on peripheral nerve remyelination and gait function for 5 weeks in a sciatic nerve crush model.

Material and methods. In total, we divided 97 rats into 5 groups: group 1 - a healthy negative control group; group $2-3$ weeks after sciatic nerve crush and 3 sessions of ESWT; group $3-5$ weeks after crush injury with 3 sessions of ESWT; group $4-3$ weeks after crush injury with no ESWT; and group 5 - 5 weeks after crush injury with no ESWT. The focused ESWT was applied to the unilateral sciatic nerve injury site. One session consisted of 1,500 stimuli, and the session were performed at intervals of 1 week.

Results. The degree of myelination and expression of myelin basic protein at the distal part of the injured sciatic nerve tended to increase in the ESWT groups compared with the no-ESWT groups 3 and 5 weeks after crush injury. Regarding the functional gait recovery, the print width and area of the injured leg in the ESWT groups was significantly larger than that in the no-ESWT groups 3 and 5 weeks after crush injury.

Conclusions. The ESWT may enhance peripheral nerve remyelination and gait function in a nerve crush model. Long-term follow-up after ESWT and investigation of molecular mechanisms will be needed to confirm these therapeutic effects.

Key words: peripheral nerve injuries, extracorporeal shockwave therapy, myelin basic protein, remyelination, gait 


\section{Introduction}

Trauma or entrapment, herniated intervertebral discs, and cancer metastasis can cause damage to the peripheral nervous system, resulting in various neuromuscular disorders, depending on the location and extent of the lesion. These conditions present focal neuropathy, plexopathy and radiculopathy, all of which are associated with neuropathic pain and muscle paralysis. The clinical importance of peripheral neuropathy is increasing because of the rising number of neuromuscular diseases due to aging of the society. The aim of treatment is to improve the function and quality of life, in patients with peripheral neuropathy.

Inflammatory reactions occur as macrophages invade the tissue surrounding damaged peripheral nerves. Next, Wallerian degeneration, demyelination and axon regeneration processes are initiated, with various signaling pathways involved. During this process, Schwann cells play a key role in orchestrating repair. ${ }^{1-3}$ Extracorporeal shockwave therapy (ESWT) has long been used in urinary stone lithotripsy treatment. In the musculoskeletal field, it has been widely used and researched in lateral and medial epicondylosis, plantar fasciitis, calcific tendinitis of the shoulder, and bony union after fracture. Recently, it has been suggested that these indications should be expanded to include muscle rigidity, muscular pain, knee degenerative arthritis, and lymphedema. ${ }^{4}$

Peripheral nerves regenerate slowly in the human tissues. Various conservative treatments such as electrical stimulation and steroid injection have been used in an attempt to alleviate symptoms after peripheral nerve injury, but without significant results. Recently, stem cell injection has been proven to be effective in animal studies of peripheral nerve injury, but its safety has not been established for long-term follow-up in clinical practice. Thus, noninvasive ESWT may be a realistic alternative. However, the effects of ESWT on angiogenesis, metabolic activation and inflammation response have been suggested but remain to be established. In addition, stimulation parameters such as optimized stimulation intensity $\left(\mathrm{mJ} / \mathrm{mm}^{2}\right)$, and number and duration of treatment sessions are also different and remain controversial between studies. Although the efficacy and safety of ESWT have been established, studies on the mechanism of effect are essential in order to expand clinical applications.

The ESWT is thought to cause molecular and biological changes, in addition to mechanical and physical stimulation by shockwave, but it is difficult to determine which among various signaling pathways is involved. It has previously been shown that adipose-derived stem cells maintain pluripotency when ESWT is applied and promote differentiation into Schwann-like cells. ${ }^{5}$ It has also been shown that ESWT induces the proliferation of bone marrow stromal cells and their differentiation into osteoprogenitors via induction of TGF- $\beta 1 .^{6}$ Recent animal studies have also shown that ESWT stimulates peripheral nerve regeneration. ${ }^{7-8}$

Although ESWT is actively used to treat various diseases other than peripheral nerve damage, its therapeutic effects need to be investigated through animal experiments to validate clinical use and study. We investigated the effects of ESWT on peripheral nerve remyelination and gait function for 5 weeks in a sciatic nerve crush model.

\section{Material and methods}

\section{Animal model}

Nine-week old male Sprague Dawley rats were anesthetized with isoflurane inhalation, and were subject to right sciatic nerve crush proximal to the bisection area with forceps for $40 \mathrm{~s}$. After the crushed nerve was released, the sciatic nerve remained compressed and became pale (Fig. 1). A single endoneurial suture was conducted using 8-0 nylon in order to identify the site of injury when collecting tissue. After the rat recovered from general anesthesia, acetaminophen syrup was administered orally to relieve postoperative pain. Ninety-seven rats were divided into 5 groups: group 1 - healthy group as a negative control; group - 3 weeks after crush injury with 3 sessions of ESWT; group $3-5$ weeks after crush injury with 3 sessions of ESWT; group $4-3$ weeks after crush injury with no ESWT applied; and group 5 - 5 weeks after crush injury with no ESWT. The study protocol was reviewed and approved by the Institutional Animal Care and Use Committee of Chungnam National University Hospital, Daejeon, South Korea.

\section{Extracorporeal shockwave therapy}

The focused ESWT device (Dornier MedTech, Weßling, Germany), which is widely used in clinical fields, was applied only to the unilateral sciatic nerve injury site. One session consisted of 1,500 stimuli, and the sessions were performed at intervals of 1 week. Peripheral nerves and surrounding tissues were collected from different animals at 1 week and 3 weeks after the 3 sessions of ESWT were completed.

\section{Immunostaining}

Immunofluorescence was performed on tissue collected 3 or 5 weeks after crush injury. The rats were anesthetized with sodium pentobarbital (50 mg/ kg, intraperitoneal) and perfused transcardially with heparinized phosphatebuffered saline (PBS, pH 7.4), followed by perfusion with $4 \%$ paraformaldehyde for $15 \mathrm{~min}$. The sciatic nerve from the injured site was removed immediately. Cryoblocks were sectioned and immunostained with anti-myelin basic protein (MBP) primary antibody (1:400; \#MAB386, Merck Millipore, Burlington, USA). 


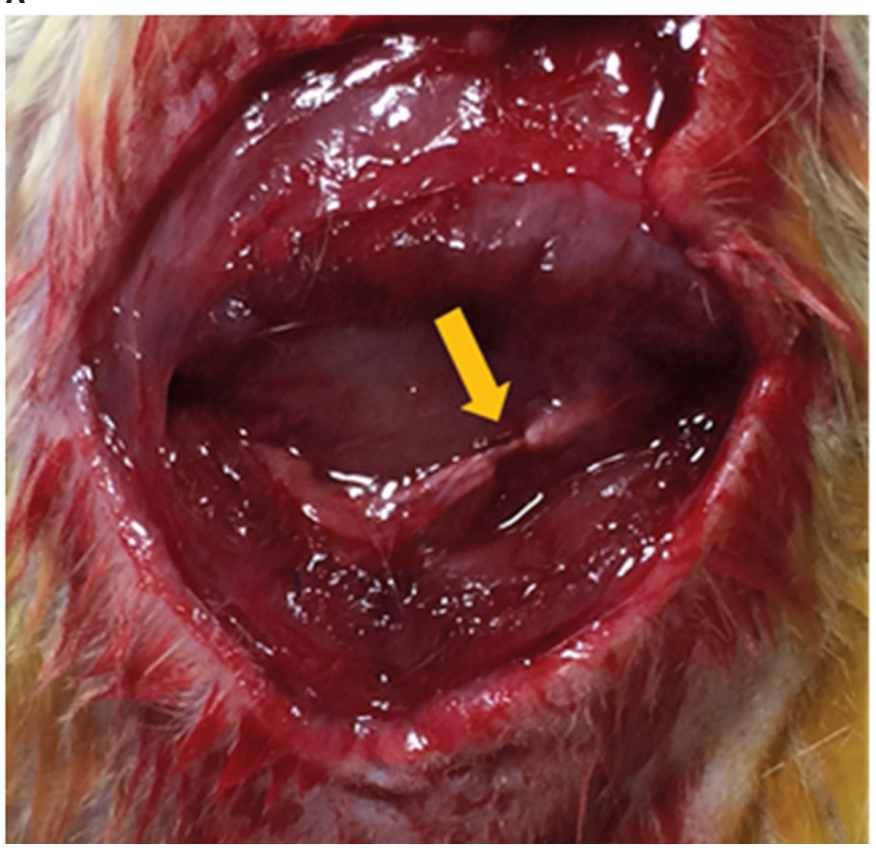

B

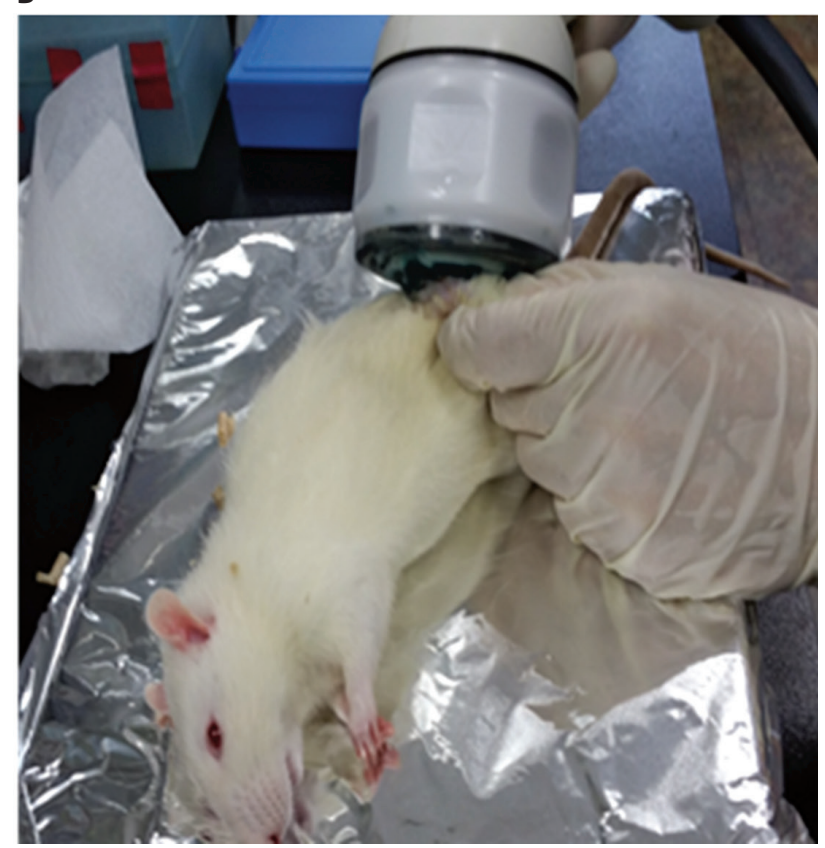

Fig. 1. Sciatic nerve crush and application of ESWT. A. When sciatic nerve crush was applied to the proximal portion of the sciatic nerve for 40 s, the nerve became compressed and pale (arrow). B. ESWT was applied to the sciatic nerve injury site

\section{Electron microscopy}

The sciatic nerve was removed and stored in the fresh fixative solution overnight at $4^{\circ} \mathrm{C}$. Tissues were washed in $0.1 \mathrm{M}$ phosphate buffer, post-fixed in $1 \%$ osmium tetroxide for $2 \mathrm{~h}$ and dehydrated through an ascending series of ethanol and propylene oxide, and then embedded in Epon812 mixture (Oken Shoji, Tokyo, Japan). Semi-thin sections ( $\times 200$ magnification) were made using Leica EM UC7 ultramicrotome (Leica Microsystems, Wetzlar, Germany), mounted on \#200 mesh copper grids, stained with $2 \%$ uranyl acetate and Reynold's lead citrate for 5 min each, and observed under a Hitachi H-7650 transmission electron microscope (Hitachi, Tokyo, Japan) at the accelerating voltage of $80 \mathrm{kV}$.

\section{Quantitative polymerase chain reaction}

Since the tissue volume of the rat sciatic nerve is low, right sciatic nerves of 3 rats were harvested and pooled. Total RNA was extracted from the sciatic nerve tissue using TRIzol reagent (Invitrogen, Carlsbad, USA) according to the manufacturer's instructions. After purification with a RNA isolation kit (Hybrid-R; GeneAll Biotechnology, Seoul, South Korea; 305-101) the concentration and purity of RNA were assessed using the NanoDrop spectrophotometer (Thermo Fisher Scientific, Waltham, USA).

Total RNA was used for cDNA synthesis with a TOPscript cDNA synthesis kit (Enzynomics, Daejeon, South Korea; RT 220) in a $20 \mu \mathrm{L}$ reaction. Subsequently, quantitative polymerase chain reaction (qPCR) was performed in duplicate in a total volume of $10 \mu \mathrm{L}$, including each 5 pM primer, cDNA and TOPreal qPCR SYBR mix (Enzynomics; RT 500). The qPCR conditions were $95^{\circ} \mathrm{C}$ for $10 \mathrm{~min}$, then 40 amplification cycles of $95^{\circ} \mathrm{C}$ for $15 \mathrm{~s}$ and $60^{\circ} \mathrm{C}$ for $1 \mathrm{~min}$ (AriaMx Realtime PCR System; Agilent Technologies Santa Clara, USA). The primer sequences (Cosmogenetech, Daejeon, South Korea) used for qPCR were as follows: rat GAPDH, forward: 5'- CTC ATG ACC ACA GTC CAT GC -3', reverse: 5'- TTC AGC TCT GGG ATG ACC TT -3'; rat MBP forward 5'-agagaccetcacagcgacac-3', reverse 5'-agggagccgtagtgggtagt-3'. The mRNA expression level was normalized to GAPDH, and the relative mRNA expression was calculated using the $2^{-\Delta \Delta C t}$ method, as previously described. ${ }^{9}$

\section{CatWalk-automated gait analysis}

We performed gait analysis using the CatWalk XT system (Noldus Information Technology, Wageningen, the Netherlands) as a functional evaluation after 1 week of adaptation. Print width $(\mathrm{cm})$ and area $\left(\mathrm{cm}^{2}\right)$ of the right leg were divided by those of the left leg at 3 and 5 weeks respectively after crush injury to derive a ratio of print width and area between both legs.

\section{Statistics}

Statistical analyses were carried out with PASW Statistics v. 18 software (SPSS Inc., Chicago, USA). The MannWhitney $U$ test was used to compare differences in qPCR 
results. The independent t-test and analysis of variance (ANOVA) were used to compare differences in CatWalk data at 3 and 5 weeks each. Differences were considered significant at a p-value $<0.05$.

\section{Results}

\section{Degree of myelination}

The distal part of the injured sciatic nerve was prepared and observed. In transmission electron microscopy $(\times 3,000$ magnification) and immunofluorescence staining, the degree of myelination tended to increase in the ESWT stimulation groups compared to the no-ESWT groups 3 and 5 weeks after crush injury (Fig. 2A,B). In qPCR, expression of MBP tended to be higher in the ESWT groups ( $\mathrm{n}=18)$ than no-ESWT groups $(\mathrm{n}=18)$ when shown using the pooled average method (Fig. 2C).

\section{Functional gait recovery}

In the right sciatic nerve crush model, we performed gait analysis using the CatWalk apparatus as a functional evaluation. Three weeks after crush injury, the print width and print area (\%) of the ESWT groups were significantly larger than in the no-ESWT groups $(\mathrm{p}=0.030$ for width and $\mathrm{p}=0.003$ for area). This improvement was maintained 5 weeks after crush injury $(\mathrm{p}=0.009$ for width and $\mathrm{p}=0.006$ for area) (Fig. 3). This indicates that gait recovery was achieved when ESWT was applied after sciatic nerve injury.

\section{Discussion}

Microsurgical repair has been performed in cases of traumatic or postoperative peripheral nerve transection, but is not applicable to partial neuronal injury. Electrical stimulation has also been used to prevent muscle atrophy caused by peripheral nerve injury, but is not effective on promoting neural recovery in severe injury.

Stem cell application has recently been suggested as a potential innovative treatment. ${ }^{10}$ Stem cells are being tested for amyotrophic lateral sclerosis and serious central nervous system disorders such as stroke and spinal cord injury, and are not commercially available for peripheral neuropathy due to possible long-term adverse effects. The PTEN blockers and microRNA-222 have been studied in animals, ${ }^{11,12}$ but they have not been used in the clinical
A

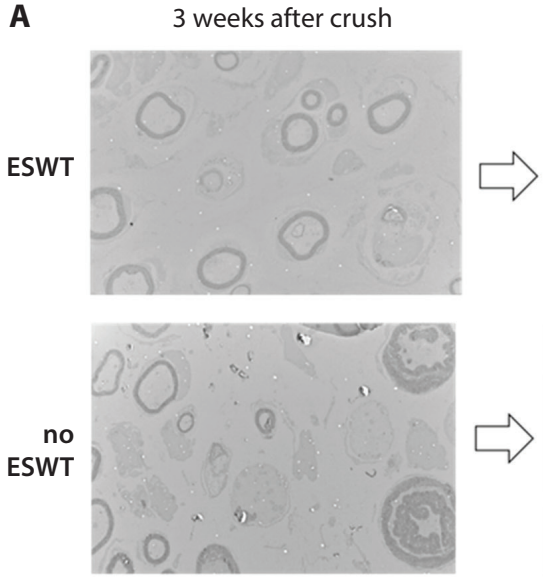

B 3 weeks after crush
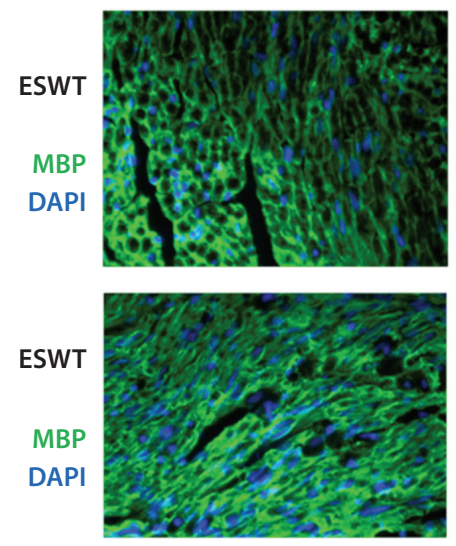

5 weeks after crush
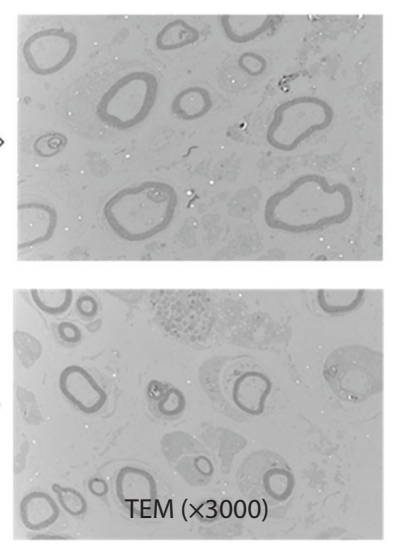

5 weeks after crush
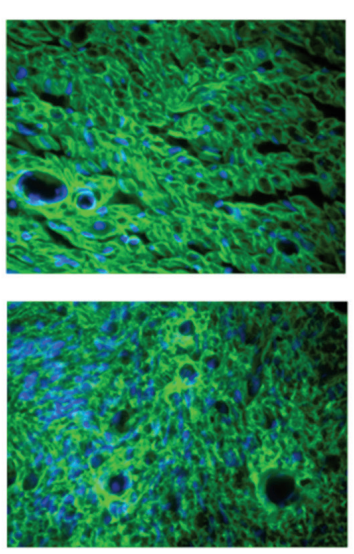

$\times 400$

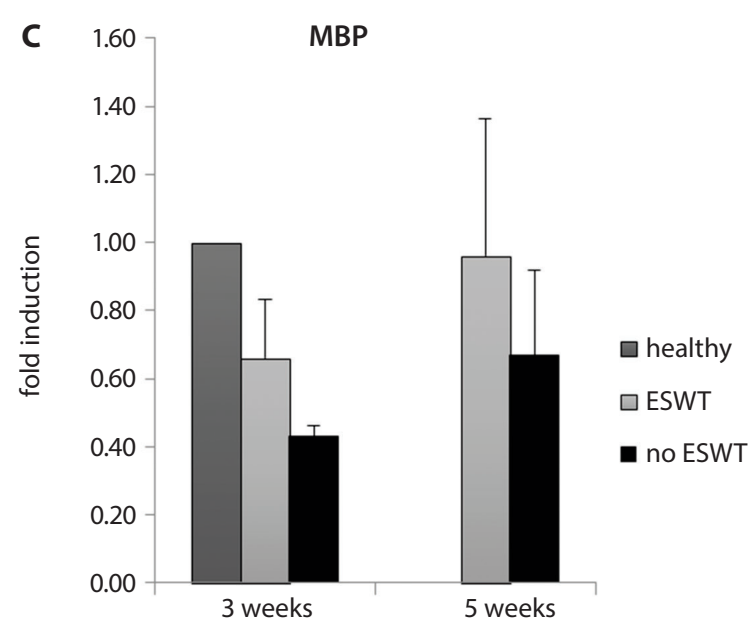

Fig. 2. Expression of myelin basic protein. A and B. In transmission electron microscopy (A) and immunofluorescent staining (B), the degree of myelination increased in the ESWT stimulation groups compared to the no-ESWT groups after 3 weeks and 5 weeks after crush injury. C. In qPCR, expression of myelin basic protein (MBP) tended to be higher in ESWT groups than in the no-ESWT groups by pooled average 

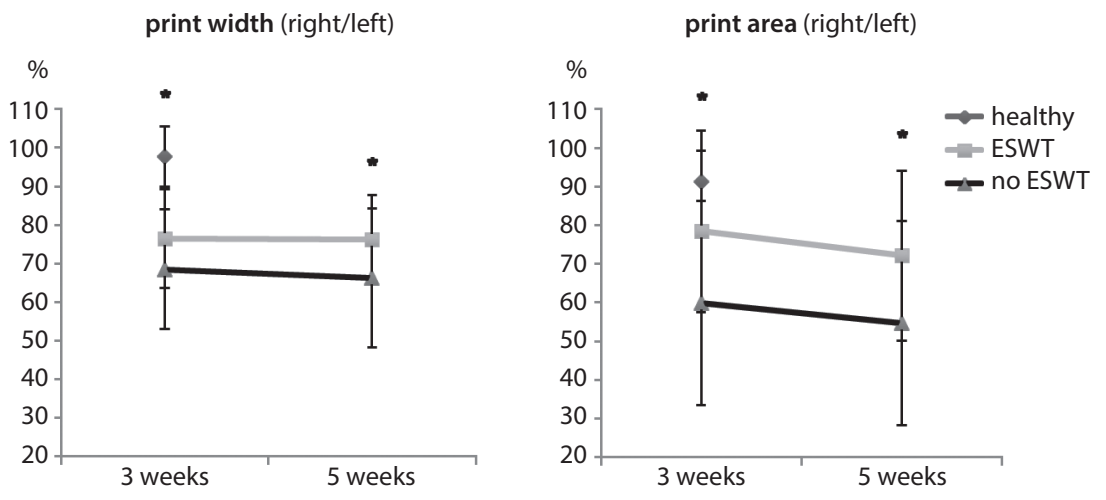

Fig. 3. Gait analysis using CatWalk. Three weeks after crush injury, the ESWT group had significantly larger print width and print area (\%) than the no-ESWT group ( $p=0.030$ for width and $p=0.003$ for area). This improvement was maintained 5 weeks after crush injury $(p=0.009$ for width and $p=0.006$ for area) field. Therefore, there is a need to develop a realistic and non-adverse treatment modality that can be immediately applied to patients with peripheral nerve damage causing functional decline and poor quality of life.

Recent studies have shown that Schwann cells are the major expression cells of GDF15, and may be involved in the regeneration of peripheral nerves through increases in the myelination of injured peripheral nerves or through improving nerve conduction velocity. ${ }^{13-15}$ However, in vivo experiments focused on the mechanisms have not been performed. Schwann cells are an important cell type involved in the myelination of peripheral nerves, and Schwann cell plasticity and de-differentiation are newly suggested restoration mechanisms after injury. In normal conditions, Schwann cells are in a resting state that has no other function except for its basic role of nerve conduction. However, when the peripheral nerve is damaged, Schwann cells maintain the function of neuronal cells. This is a substantial phenotypic transformation of Schwann cells, referred to as plasticity or de-differentiation. ${ }^{16}$ Schwann cell de-differentiation is largely divided into 2 mechanisms. Firstly, it participates in the demyelination process by activating lysosome or protease proteins. Secondly, it secretes neurotrophic factors involved in peripheral nerve survival or axonal regeneration.

It is also known that the neurotrophic factors secreted during Schwann cell de-differentiation are glial cell-derived neurotrophic factor (GDNF) and extracellular matrix proteins. These factors influence the survival of motor neurons and dorsal root ganglion in the spinal cord, and signal transduction through c-jun is involved in axonal regeneration. These factors and the relationship between GDF15 and Krox20 in the peripheral nerves have not been elucidated. In this study, induction of Schwann cell plasticity could be suggested as a mechanism of peripheral nerve remyelination and functional recovery.

We demonstrated that degree of myelination and expression of MBP tended to increase in the ESWT groups compared to the no-ESWT groups 3 and 5 weeks after crush injury. Regarding functional gait recovery, the print width and areas of the injured leg in the ESWT groups were significantly larger than those in the no-ESWT groups measured 5 weeks after crush injury. Considering these findings, ESWT may enhance gait function through peripheral nerve remyelination in a crush model.

There could be several limitations in this study. First, toxin-induced demyelination would be more suitable than nerve crush for pure demyelination without axonal loss. However, we selected the crush model that is similar to clinical situation for peripheral nerve injury. Second, the ESWT applicator was relatively large for the lower limb of adult rats. Thus, we tried to focus the center of the applicator on the sciatic nerve crush site. Third, different individuals were analyzed at 3 and 5 weeks after crush injury because rats were sacrificed and tissues harvested at 3 and 5 weeks, respectively. Although longitudinal follow-up in the same individuals would be more reliable to interpret the results, it is not possible in animal studies. Long-term follow-up after ESWT with a small applicator suitable for rats will be needed to confirm the therapeutic effects. Furthermore, molecular mechanisms regarding various signaling pathways are yet to be investigated.

\section{ORCID iDs}

Hyun Jung Park (10 https://orcid.org/0000-0002-1982-9172 Jinpyo Hong (1) https://orcid.org/0000-0002-4776-4396 Yibo Piao (10) https://orcid.org/0000-0001-9628-4906 Hyo Jung Shin (1) https://orcid.org/0000-0002-3530-101X Se Jeong Lee (1) https://orcid.org/0000-0002-4437-8270 Im Joo Rhyu (1) https://orcid.org/0000-0002-5558-6278 Min-Hee Yi (D) https://orcid.org/0000-0003-2066-3584 Jinhyun Kim (1) https://orcid.org/0000-0001-5235-2612 Dong Woon Kim (1) https://orcid.org/0000-0001-7691-1394 Jaewon Beom (10) https://orcid.org/0000-0001-7984-9661

\section{References}

1. Morton PD, Johnstone JT, Ramos AY, Liebl DJ, Bunge MB, Bethea JR. Nuclear factor-KB activation in Schwann cells regulates regeneration and remyelination. Glia. 2012;60(4):639-650.

2. Glenn TD, Talbot WS. Signals regulating myelination in peripheral nerves and the Schwann cell response to injury. Curr Opin Neurobiol. 2013;23(6):1041-1048.

3. Martini R, Fischer S, López-Vales R, David S. Interactions between Schwann cells and macrophages in injury and inherited demyelinating disease. Glia. 2008;56(14):1566-1577.

4. Bae H, Kim HJ. Clinical outcomes of extracorporeal shock wave therapy in patients with secondary lymphedema: A pilot study. Ann Rehabil Med. 2013;37(2):229-234. 
5. Schuh CM, Heher P, Weihs AM, et al. In vitro extracorporeal shock wave treatment enhances stemness and preserves multipotency of rat and human adipose-derived stem cells. Cytotherapy. 2014; 16(12):1666-1678.

6. Hausner T, Pajer K, Halat G, et al. Improved rate of peripheral nerve regeneration induced by extracorporeal shock wave treatment in the rat. Exp Neurol. 2012;236(2):363-370.

7. Wu YH, Liang HW, Chen WS, Lai JS, Luh JJ, Chong FC. Electrophysiological and functional effects of shock waves on the sciatic nerve of rats. Ultrasound Med Biol. 2008;34(10):1688-1696.

8. Wang FS, Yang KD, Chen RF, Wang CJ, Sheen-Chen SM. Extracorporeal shock wave promotes growth and differentiation of bone-marrow stromal cells towards osteoprogenitors associated with induction of TGF-beta1. J Bone Joint Surg Br. 2002;84(3):457-461.

9. Livak KJ, Schmittgen TD. Analysis of relative gene expression data using real-time quantitative PCR and the 2(-delta delta $C(T)$ ) method. Methods. 2001;25(4):402-408.

10. Lee EJ, Xu L, Kim GH, et al. Regeneration of peripheral nerves by transplanted sphere of human mesenchymal stem cells derived from embryonic stem cells. Biomaterials. 2012;33(29):7039-7046.
11. Zhou S, Shen D, Wang Y, et al. microRNA-222 targeting PTEN promotes neurite outgrowth from adult dorsal root ganglion neurons following sciatic nerve transection. PLoS One. 2012;7(9):e44768.

12. Wu D, Murashov AK. Molecular mechanisms of peripheral nerve regeneration: Emerging roles of microRNAs. Front Physiol. 2013;4:55.

13. Mensching L, Börger AK, Wang X, Charalambous $P$, Unsicker $K$, HaastertTalini K. Local substitution of GDF-15 improves axonal and sensory recovery after peripheral nerve injury. Cell Tissue Res. 2012;350(2): 225-238.

14. Pereira JA, Lebrun-Julien F, Suter U. Molecular mechanisms regulating myelination in the peripheral nervous system. Trends Neurosci. 2012;35(2):123-134.

15. Hung HA, Sun G, Keles S, Svaren J. Dynamic regulation of Schwann cell enhancers after peripheral nerve injury. J Biol Chem. 2015;290(11): 6937-6950.

16. Jessen KR, Mirsky R. Negative regulation of myelination: Relevance for development, injury, and demyelinating disease. Glia. 2008;56(14): 1552-1565. 